\title{
Les médecins suisses: un groupe de contrôle?
}

\section{Yvonne Gilli}

Dr méd., membre du Comité central de la FMH, responsable du département Numérisation/eHealth

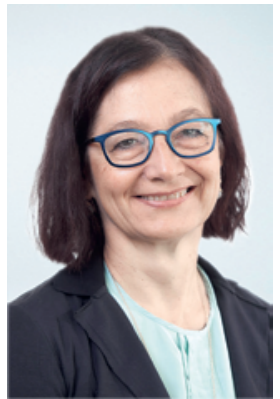

L'étude SISA publiée en 2015 présente une image plutôt sombre de la numérisation au sein du corps médical ambulatoire [1], bien que le degré de numérisation augmente dans les cabinets médicaux suisses, selon une enquête annuelle de l'OCDE. La part de médecins qui utilisent des dossiers patients informatisés est passée à 50\% en 2018 [2]. L'étude SISA rappelle aussi que les médecins en Suisse font partie d'une expérimentation globale: même si le système de santé de notre pays est comparable aux systèmes canadien ou américain pour son organisation ou sous l'aspect des principes de l'économie de marché, il ne connaît, inversement à eux, aucun incitatif visant à améliorer l'acceptation et l'utilisation de solutions informatiques dans le domaine de

La numérisation va bien au-delà du seul dossier électronique du patient.

la santé ou à promouvoir la cybersanté. Le corps médical suisse joue donc en quelque sorte le rôle de groupe de contrôle qui fait avancer la numérisation sans que l'Etat n'instaure de système d'incitatifs. Les résultats d'une étude de la FMH sur la numérisation de la prise en charge médicale du point de vue du médecin (dans le présent numéro, pages 1685-1689) attestent d'une attitude sur le fond positive et d'une ouverture du corps médical pour les services numériques de santé. Cependant, cette étude de la FMH ne laisse pas non plus le moindre doute sur le fait que les inquiétudes du corps médical quant à la numérisation doivent être prises au sérieux et qu'il est primordial d'associer les médecins à la mise en œuvre de la cybersanté pour en garantir le succès. En parallèle, il faut encourager l'interopérabilité des systèmes pour qu'elle devienne une condition nécessaire à un processus de numérisation constant dans le domaine de la cybersanté, qui inclut bien évidemment aussi l'informatique au cabinet médical, sans oublier de créer des incitatifs. Cette année, le département Numérisation/eHealth a repris les travaux relevant de l'informatique au cabinet médical, lancés initialement par l'Institut pour l'informatique au cabinet médical. Il y a quelques semaines, le département a organisé un congrès sur cette thématique dans le but de passer en revue l'avancée de la numérisation sur la base d'exemples concrets sur le terrain et de solliciter les échanges entre le corps médical et l'industrie. ${ }^{1}$ Les présentations et les exposés ont souligné que la numérisation allait bien au-delà du seul dossier électronique du patient. L'informatisation des processus au cabinet médical échoue souvent sur des choses apparemment banales, comme les échanges de données entre les applications de différents domaines tels que la comptabilité, les commandes ou la gestion de la qualité. L'absence d'intégration et d'interopérabilité rompt très rapidement la continuité des processus au cabinet médical et, manifestement, nous en sommes encore au début de la recherche de solution: l'utilisabilité des systèmes informatiques médicaux n’a guère évolué ces dernières années, comme le rappelle un sondage national réalisé régulièrement en Finlande [3].

Le manque d'incitatifs s'est également traduit par un faible déploiement du dossier médical partagé (DMP) en France [4]. Cinq ans après son lancement, seul 1,5\% de la population a ouvert un DMP et moins de $1 \%$ des

Pour réussir la mise en œuvre de la cybersanté, il est primordial d'associer les médecins et de développer un système d'incitatifs.

médecins du secteur privé participe au DMP. Du côté des médecins, ce manque d'intérêt réside dans les coûts d'investissement pour un système offrant une interface compatible avec le DMP. Ce n'est que dans une deuxième phase que le programme a été fondamentalement modifié avec la mise en place d'un système d'incitatifs. Le nombre de dossiers a alors aug menté de $50 \%$ au cours des dix premiers mois après le lancement du nouveau DMP. Reste à espérer que les médecins suisses franchiront le pas de groupe de contrôle et deviendront un groupe d'intervention.

\section{Références}

Les références se trouvent sous www.bullmed.ch $\rightarrow$ Numéro actuel ou $\rightarrow$ Archives $\rightarrow 2018 \rightarrow 48$. 


\section{Références}

1 Djalali S, Ursprung N, Rosemann T, Senn O, Tandjung R. Undirected health IT implementation in ambulatory care favors paper-based workarounds and limits health data exchange. Int J Med Inform. 2015.

2 Golder L, Jans C. Swiss eHealth Barometer 2018, 2018.

3 Kaipio J, et al. Usability problems do not heal by themselves: National survey on physicians' experiences with EHRs in Finland. Int J Med Inform. 2017.

4 Séroussi B, Bouaud J. Adoption of a Nationwide Shared Medical Record in France: Lessons Learnt after 5 Years of Deployment. Amin Annu Symp Proc Arch. 2016. 